\title{
Targeting MicroRNAs for Personalized Cancer Therapy
}

\author{
Yiwei Li $^{\mathrm{a}}$ Aamir Ahmad $^{\mathrm{a}}$ Dejuan Kong ${ }^{\mathrm{a}}$ Bin Bao ${ }^{\mathrm{a}}$ Fazlul H. Sarkar ${ }^{\mathrm{a}} \mathrm{b}$ \\ Departments of a Pathology and ${ }^{b}$ Oncology, Barbara Ann Karmanos Cancer Institute, Wayne State University \\ School of Medicine, Detroit, Mich., USA
}

\section{Introduction}

After a long debate over several years, it is now becoming clear that cancer stem cells (CSCs) do exist in hematopoietic and solid malignant tumors. CSCs are a subpopulation of cancer cells which possess high capacities of self-renewal, multilineage differentiation, rapid proliferation and tumor initiation. CSCs are more resistant to chemotherapeutics and show a high potential for invasion and metastasis. Moreover, the survival of CSCs after the elimination of most cancer cells by chemotherapeutics is the major cause of cancer recurrence. Therefore, targeting CSCs in addition to conventional therapy may be a novel strategy for better treatment of cancer. Recently, miRNAs have received increasing attention in cancer research. miRNAs are aberrantly expressed in cancer cells. Studies on miRNAs have demonstrated that they could critically control the development, self-renewal and differentiation of CSCs as well as regulate drug sensitivity and CSC invasion and metastasis. Targeting CSC-related miRNAs could be a promising approach to eliminate CSCs and thus inhibit cancer progression.

\section{KARGER}

E-Mail karger@karger.com www.karger.com/mpp
(๑) 2013 S. Karger AG, Basel 1011-7571/13/0225-0415\$38.00/0

Open access

This is an Open Access article licensed under the terms of the Creative Commons Attribution-NonCommercial 3.0 Unported license (CC BY-NC) (www.karger.com/OA-license), applicable to the online version of the article only. Distribution permitted for non-commercial purposes only.

\section{CSC-Related miRNAs}

It is important to note that the miRNA expression patterns in CSCs from different types of cancers are distinct. CSCs from various cancers may have some miRNAs in common; however, they also express distinct miRNAs dependent on context. In breast cancer, CSCs with CD $44^{+}$ and $\mathrm{CD} 24^{-/ l o}$ have aberrant expression of miRNAs and much greater self-renewal activity, proliferation and tumor initiation than other cancer cells. The high expression of miR-21, miR-181a and miR-181b has been observed in breast CSCs; however, these cells express very low levels of let-7, miR-34, miR-200, miR-29, miR-30a and miR-93.

Similar to breast CSCs, prostate CSCs express a very low level of miR-34, which targets AR and CD44 expression. Therefore, both breast and prostate CSCs have a high expression of CD44. However, prostate CSCs express low levels of other miRNAs, different from those expressed by breast CSCs. The expression of miR-320 and miR-708 is significantly downregulated in prostate CSCs, unlike in breast CSCs. Molecular mechanistic studies show that miR-320 and miR-708 could inhibit tumor formation from CSCs by targeting $\beta$-catenin or 
AKT2 [1]; this suggests the inhibitory effects of miR-320 and $\mathrm{miR}-708$ on prostate CSCs.

In colorectal CSCs, miR-21, miR-155, miR-192, miR194 and miR-17-92 clusters are highly expressed. However, the expression levels of miR-93, miR-31, miR-221 and $\mathrm{miR}-451$ are low, suggesting the suppressive effects of these miRNAs on colorectal CSCs. To support this notion, experimental study has shown that enforced expression of miR-451 suppresses colorectal CSC self-renewal, tumor formation and chemoresistance to irinotecan [2].

Similar to colorectal and breast CSCs, the expression of the miR-17-92 cluster is upregulated and the let-7 expression is decreased in lung CSCs. Pancreatic CSCs have a high expression of miR-21 and a low expression of miR-34, miR-200 and let-7, similar to the miRNA expression patterns of breast and prostate CSCs. In other words, there are several common miRNAs expressed in the same pattern in CSCs from different types of cancers. These miRNAs could be important targets for the elimination of CSCs in general. However, the specific miRNAs altered exclusively in CSCs from one type of cancer could also be critical for the control of specific phenotypes of CSCs. Therefore, by targeting these important specific miRNAs, the complete elimination of CSCs is perhaps feasible.

\section{The Advantage of Personalized Cancer Therapy}

It is well known that cancer cells possess heterogeneous features with diverse morphologies, differential molecular markers, various clinical syndromes and distinct responses to cancer therapeutics. The genetic and epigenetic alterations and the different molecules altered in cancer cells contribute to the heterogeneity of cancers. As discussed in the previous section, patterns of miRNA expression vary in different CSCs. Even in the same type of CSC, the expression profiles of miRNAs are not identical. Because of the heterogeneity of cancers, cancer therapy should be personalized to target specific molecular markers to inhibit cancer for each patient effectively. By targeting the altered signaling pathways or miRNAs, the tumor-causing genes, miRNAs and proteins could be neutralized and the functional molecules (including mRNA, miRNA and proteins) could be modulated.

To design and conduct a personalized cancer therapy, it is necessary to obtain the profiles of molecular markers including miRNAs from patient specimens. It is also important to obtain miRNA signatures of CSCs from each patient in order to design clinical trials for personalized cancer therapy. Clinical trials were recently performed to determine the benefits of personalized cancer therapy $[3,4]$. In a trial involving 1,444 patients at the MD Anderson Cancer Center, personalized cancer therapy showed a higher overall response rate, a longer time-to-treatment failure and longer survival when compared with prior systemic therapy [4]. These exciting results suggest the beneficial effects and advantage of personalized cancer therapy. However, many more clinical trials are needed before the personalized therapy could become the standard of care in cancer therapy.

\section{Targeting Specific miRNAs for Personalized Cancer Therapy}

Several therapeutic strategies have been designed for targeting specific miRNAs in CSCs. These include synthesized oligonucleotide delivery, synthetic agent treatment and natural compound administration to target CSC-related miRNAs. Synthesized oligonucleotide delivery by lipid-based formulations or nanoparticles is more specific for targeting individual miRNA than other strategies. Based on the information from miRNA profiles of CSC, anti-sense nucleotides could be used to inhibit overexpressed CSC-specific miRNAs while sense nucleotides could be delivered to introduce CSC-suppressive miRNAs. Experimental studies have shown the successful delivery of anti-sense oligonucleotides against miR-192, miR-194 and miR-155, which are highly expressed in CSCs, for silencing these miRNAs [5]. Sense oligonucleotides have been delivered to introduce miR-34a and miR-145, which are downregulated in CSCs. The miR145 delivery reduces CSC markers, increases drug sensitivity and inhibits tumor growth and metastasis. These results demonstrate that targeting specific miRNAs with synthetic oligonucleotide could be useful for personalized cancer therapy.

Synthetic and natural agents could also regulate the expression of miRNAs. However, such regulation may not target just one specific miRNA. The first synthesized agent for targeting CSCs is salinomycin which has a high capacity for killing CSCs. In addition, novel platinum and palladium complexes have been synthesized for targeting CSCs. These agents could inhibit CSCs through miRNAmediated cellular signaling. Natural agents could also alter the levels of miRNAs. We found that natural agents such as isoflavone, 3,3'-diindolylmethane and CDF (cur- 
cumin analogue with greater bioavailability) could downregulate miR-21 expression and upregulate the expression of miR-34a, miR-146a, miR-200 and let-7, leading to the inhibition of CSC function [6]. Therefore, these natural agents could be utilized for targeting miRNAs toward personalized cancer therapy.

\section{Conclusion}

Emerging evidence suggests that targeting aberrantly expressed miRNAs in CSCs by oligonucleotides and synthetic or natural agents could become a novel strategy for realizing the dream of personalized cancer therapy. By eliminating CSCs, the inhibition of cancer drug resistance, invasion and metastasis could be achieved, which will lead to a better treatment outcome of cancer patients.

\section{References}

1 Saini S, Majid S, Shahryari V, et al: miRNA708 control of CD44(+) prostate cancer-initiating cells. Cancer Res 2012;72:3618-3630.

$>2$ Bitarte N, Bandres E, Boni V, et al: MicroRNA-451 is involved in the self-renewal, tumorigenicity, and chemoresistance of colorectal cancer stem cells. Stem Cells 2011;29: 1661-1671.
Printz C: Personalized medicine benefits patients in early trials: matching targeted therapies with gene mutations is associated with better outcomes, even in phase 1 studies. Cancer 2012;118:4911-4912.

4 Tsimberidou AM, Iskander NG, Hong DS, et al: Personalized medicine in a phase I clinical trials program: the MD Anderson Cancer Center initiative. Clin Cancer Res 2012;18: 6373-6383.
Krutzfeldt J, Rajewsky N, Braich R, et al: Silencing of microRNAs in vivo with 'antagomirs'. Nature 2005;438:685-689.

6 Bao B, Azmi AS, Ali S, et al: The biological kinship of hypoxia with CSC and EMT and their relationship with deregulated expression of miRNAs and tumor aggressiveness. Biochim Biophys Acta 2012;1826:272-296. 